\title{
Pattern ERG as a predictor in ocular hypertensive
}

\author{
Paaraj Dave, Jitendra Jethani \\ Dr. Thakorbhai V Patel Eye Institute, Vadodara, India
}

\begin{abstract}
Purpose: To report the repeatability of Pattern Electrotretinogram (PERG) and its findings in ocular hypertension (OHT) and normal eyes.

Methods: A cross-sectional study where PERG responses were compared between the study group comprising of 80 eyes of 80 patients with intraocular pressure $(I O P)>21 \mathrm{mmHg}$ and the control group with 80 eyes of 80 normal individuals $(I O P \leq 21 \mathrm{mmHg})$. The optic disc and the visual fields were normal with a visual acuity of $\geq 0.8$ in both groups. PERG was recorded twice for each individual in the control group by a single operator on 2 consecutive days to assess the repeatability.

Results: The mean age in the study and control groups was $50.05 \pm 10.03$ and $54.8 \pm 7.66$ years ( $p=0.44$ ) respectively. The mean IOP was $26.55 \pm 3.9 \mathrm{mmHg}$ for the study group as opposed to $14.45 \pm 2.9 \mathrm{mmHg}$ for the controls $(p<0.001)$. The central corneal thickness and the cup-disc ratio was similar between the groups ( $p>.05)$. The P50-N95 amplitude $(p=0.01)$ and the P50 latency $(p<0.001)$ was statistically significantly different between both the groups. The intra-class correlation coefficient (ICC) showed poor agreement for all parameters except for N35-P50 0.8 to 16 degree check size amplitude ratio (PERG ratio). Conclusion: Increase in P50 latency emerges as a new candidate for early glaucoma indicator in addition to reduction in P50-N95 amplitude. PERG parameters suffer from high test-retest variability. Deterioration in PERG recordings should be interpreted with caution. The variability is lesser for the PERG ratio which maybe more meaningful while monitoring for change over time.
\end{abstract}

Keywords: Glaucoma, Ocular Hypertension, Pattern Electroretinogram, PERG, Repeatability.

\section{Introduction}

Ocular hypertension (OHT) is defined as elevated intraocular pressure (IOP) without any glaucomatous visual field defects or optic disc changes. Elevated IOP is a major modifiable risk factor for glaucoma. Glaucoma is characterized by chronic retinal ganglion cell (RGC) loss. A sizable fraction (25\%-35\%) of RGCs is already lost when visual field defects become apparent in automated visual field testing. ${ }^{1}$

The retinal response to pattern stimulation, the pattern electroretinogram (PERG), predominantly reflects RGC activity. ${ }^{2-6}$ Reduction in the PERG amplitude had been reported in patients with $\mathrm{OHT} .^{7-14}$ Knowledge of the variability of PERG is extremely important especially when we want to judge its deterioration. This variability has been studied before. ${ }^{6,15-16}$ PERG testing is essentially objective and theoretically more repeatable than subjective testing because it is minimally affected by motor response and learning effects. The aim of our study is to find whether a difference in the PERG recordings between OHT and normal patients exists and also to ascertain

Correspondence: Jitendra Jethani, Thakorbhai V Patel Eye Institute, Haribhakti Complex, Salatwada, Vadodara-390001, India.

E-mail: xethani@rediffmail.com 
that this difference is outside the test-retest variability of the PERG machine. To the best of our knowledge both these things together have not been done before.

\section{Materials and methods}

This was a cross sectional study and an informed consent was obtained from all subjects. For the study group, we included 80 eyes of 80 consecutive patients diagnosed as ocular hypertension (OHT) from October 2012 to April 2013. Inclusion criteria were: IOP (measured with Goldmann applanation tonometry at two different times) $>21 \mathrm{mmHg}$, open angles on 4-mirror gonioscopy without indentation, normal and reliable visual field test results as described below and normal optic disc at clinical examination and on photographs. Our control group also consisted of 80 eyes of 80 random patients recruited during the same period with similar age range. The inclusion criteria were similar except for the IOP which was $\leq 21 \mathrm{mmHg}$. Exclusion criteria were visual acuity $<0.8$, spherical correction outside $\pm 5.0 \mathrm{D}$, cylinder correction outside $\pm 3.0 \mathrm{D}$, secondary glaucoma (e.g., pigment dispersion or pseudoexfoliation syndrome), and diabetic retinopathy or any other condition capable of causing visual field loss or optic disc damage. Pressure lowering treatment was not an exclusion criterion. Treatment was actively recommended only to patients with $\mathrm{IOP}>28 \mathrm{mmHg}$ which is the standard practice at our institute.

A complete ophthalmic examination including best corrected visual acuity, IOP measurement with Goldmann applanation tonometer, pachymetry, gonioscopy, indirect ophthalmoscopy, slit lamp biomicroscopy, visual fields with Humphrey Visual Field Analyzer II (Carl Zeiss Meditec, Dublin, CA) and PERG recording was done. The PERG was recorded twice for each individual in the control group by a single operator $(\mathrm{JJ})$ on 2 consecutive days at approximately the same time of the day in order to find out the test-retest variability. All other examinations were performed by one of the two authors (PRD and JJ).

\section{Visual field}

All the tested eyes had a pupil diameter $\geq 3 \mathrm{~mm}$ and corrective lenses were placed on the lens holder to evaluate the visual field. Visual field examination was performed using 24-2 SITA Standard strategy by HVF analyzer. Only patients with reliable visual fields (false positive, false negative $<33 \%$, fixation losses $<20 \%$ ) were included. A normal visual field was defined by the absence of each of these responses: a cluster of 3 points lower than $p<5 \%$ or a cluster of 2 points lower than $p<1 \%$ on a pattern deviation plot, or PSD with $p<5 \%$. All visual field examinations were done not more than 1 month prior to doing the first PERG.

\section{PERG Recording}

For PERG recording, we followed the International Society for Clinical Electrophysiology of Vision (ISCEV) standard guidelines. ${ }^{17}$ RETIport 21 (version: 19-99-04-7.2E; Roland Consult, Brandenburg, Germany) machine was used to perform the PERG under photopic condition at a distance of 1 meter from the 19" monitor. The stimulus 
was a high contrast (99\%) black and white stimulus with a mean luminance of 80 $\mathrm{cds} / \mathrm{m}^{2}$ and a chequerboard size of $0.8^{\circ}$ or $16^{\circ}$.The stimulus frequency was 15.005 $\mathrm{Hz}$, cycle time of 0.6664 seconds and a plot time of 400 milliseconds. Responses from both eyes were measured simultaneously. Before testing, the electrode impedance was monitored automatically and an on-screen indicator signaled an acceptable impedance $(<10 \mathrm{kOhm})$. Retinal potentials were recorded with corneal DTL electrodes and gold cup electrodes at the outer ipsilateral canthus served as reference. ${ }^{18}$ Signals were amplified and filtered with a 2-channel bio signal amplifier. During each examination, two PERG measurements were taken, and the mean of the traces was used to yield the raw PERG amplitude. The glaucoma program calculated the PERG ratio (response amplitude to $0.8^{\circ}$ checks divided by the response amplitude to $16^{\circ}$ checks). The spectrum in the software was used for getting the P50-N95 marker. Fig. 1 and Fig. 2 show the representative PERG traces of normal control and ocular hypertensive eyes respectively.
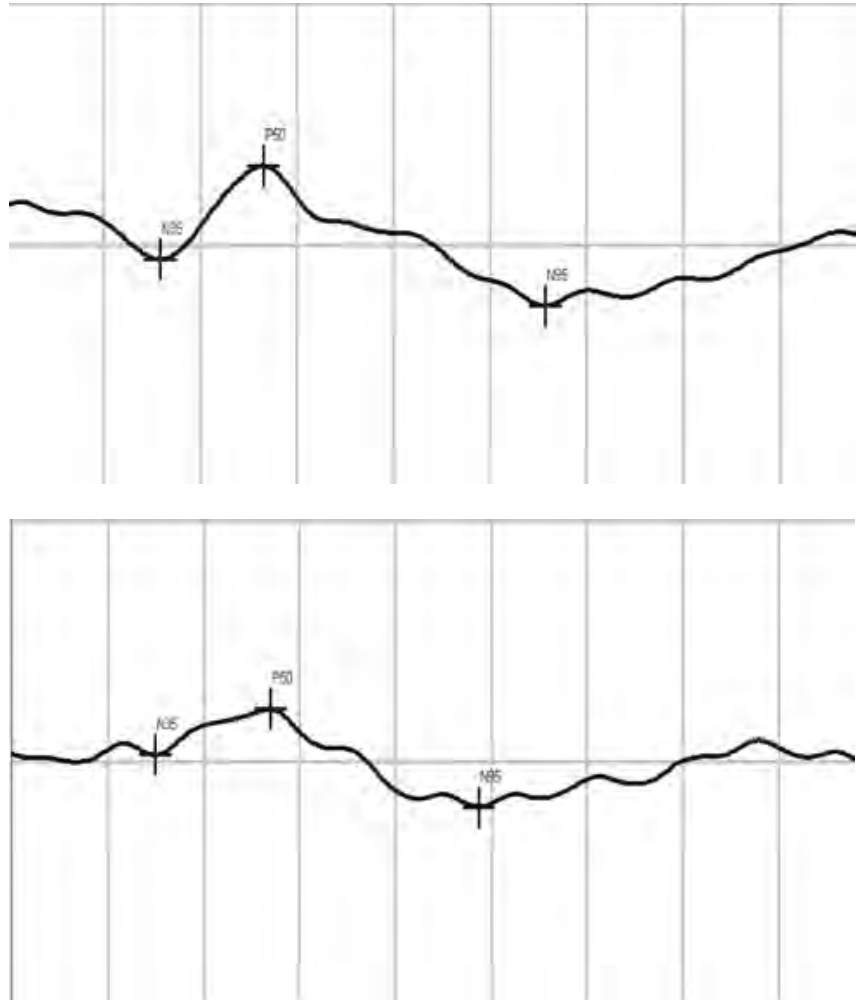

Fig. 1. A representative Pattern Electroretinogram recording of a normal control eye.
Fig. 2. A representative Pattern Electroretinogram recording of an eye with ocular hypertension.

\section{Statistical Analysis}

Descriptive and inferential statistics were performed using STATA version 12 for Windows (StataCorp LP, Texas).Mann-Whitney $U$ test for continuous variables and Chi Square test for categorical variables was used to compare the baseline 
parameters between the groups. The $95 \%$ confidence limits of variability for all PERG parameters were calculated from the Bland-Altman plot (BAP) method. The intra-class correlation coefficients (ICC) were also calculated for all PERG parameters.

\section{RESULTS}

Forty eyes of 80 patients were diagnosed during the study period with ocular hypertension. Twelve patients had a positive family history of glaucoma $\left(1^{\text {st }}\right.$ degree relative having glaucoma).Twenty-two of the 80 eyes were on topical medication. Sixteen of these were on a $\beta$-blocker and the remaining 6 on a prostaglandin analogue. Another 80 eyes of 80 patients were recruited as controls during the same period. Table 1 shows the demographic data and PERG parameters of the included patients.

Table 1. Baseline demographics and pattern electroretinogram readings

\begin{tabular}{|r|c|c|c|}
\hline Parameters & OHT & Controls & p value \\
\hline Age (years) & $50.05 \pm 10.03$ & $54.8 \pm 7.66$ & 0.44 \\
\hline Male:Female & $11: 9$ & $8: 12$ & 0.34 \\
\hline IOP (mmHg) & $26.55 \pm 3.9$ & $14.45 \pm 2.9$ & $<0.001$ \\
\hline CCT & $543.95 \pm 36.86$ & $530.5 \pm 48.9$ & 0.15 \\
\hline CDR & $0.45 \pm 0.15$ & $0.35 \pm 0.07$ & $<0.001$ \\
\hline P50-N95amp & $2.5 \pm 0.82$ & $3.55 \pm 1.29$ & $<0.001$ \\
\hline P50-N95lat & $94.5 \pm 9.02$ & $94.8 \pm 9.39$ & 0.82 \\
\hline N35lat16 & $23.62 \pm 4.6$ & $22.15 \pm 3.4$ & 0.15 \\
\hline N35lat0.8 & $34.5 \pm 3$ & $34.4 \pm 8.07$ & 0.17 \\
\hline P50lat16 & $51.9 \pm 5.74$ & $43.35 \pm 2.53$ & $<0.001$ \\
\hline P50lat0.8 & $57.7 \pm 8.2$ & $52.05 \pm 8.52$ & $<0.001$ \\
\hline N35-P50amp16 & $4.7 \pm 1.8$ & $5.93 \pm 2.87$ & 0.03 \\
\hline N35-P50amp0.8 & $2.04 \pm 1.27$ & $2.2 \pm 1.53$ & 0.99 \\
\hline PERG ratio (right) & $0.365 \pm 0.089$ & $0.284 \pm 0.183$ & 0.04 \\
\hline PERG ratio(left) & $0.397 \pm 0.278$ & $0.49 \pm 0.175$ & 0.08 \\
\hline
\end{tabular}

*P50-N95amp =P50-N95 amplitude +P50-N95lat = P50-N95 latency ‡N35lat16=N35 latency with 16 degree check size $\S N 35$ lat $0.8=$ N35 latency with 0.8 degree check size ||P50lat16 = P50 latency with 16 degree check size **P50lat $0.8=$ P50 latency with 0.8 degree check size $++N 35-P 50$ lat $16=$ N35-P50 latency with 16 degree check size $\neq \ddagger N 35-\mathrm{P} 50$ lat $0.8=\mathrm{N} 35-\mathrm{P} 50$ latency with 16 degree check size 
The age, gender and central corneal thickness was similar between the two groups. The mean IOP in the OHT group was $26.55 \pm 3.9 \mathrm{mmHg}$ compared to $14.45 \pm 2.9 \mathrm{mmHg}$ in the control group ( $\mathrm{p}<0.001)$. The P50-N95 latency (P50-N95lat), N35 latency with 16 degree check size (N35lat16), N35 latency with 0.8 degree check size (N35lat0.8) and the N35-P50 amplitude with 0.8 degree check size (N35-P50amp0.8) was similar in both the groups ( $p>0.05$ ). The P50-N95 amplitude (P50-N95amp),P50 latency with both 16 and 0.8 degree check size (P50lat16, P50lat0.8) and N35-P50 amplitude with 16 degree check size (N35-P50amp16) was statistically significantly different between the groups $(p<0.05)$ as shown in table1.

Table 2 shows the $95 \%$ confidence limits of variability for all PERG parameters calculated from Bland-Altman plots. Only P50 latency with 16 degree check size (P50lat16) had a difference beyond the 95\% confidence limits between the two groups. Table 2 also shows the ICC for the PERG parameters. The ICC showed poor agreement for all the PERG parameters except for the PERG 0.8 to 16 degree check size amplitude ratio for the right [PERG ratio (right)] and the left eye [PERG ratio (left)] which was 0.69 and 0.68 respectively.

Table 2. Test-retest variability for pattern electroretinogram parameters

\begin{tabular}{|c|c|c|c|c|c|}
\hline Parameters & $\begin{array}{c}\text { Control 1 } \\
\text { Run }\end{array}$ & $\begin{array}{c}\text { Control 2 } \\
\text { Run }\end{array}$ & p value & $\begin{array}{c}\text { Bland-Altman } \\
95 \% \text { limit }\end{array}$ & ICC \\
\hline P50-N95amp & $3.55 \pm 1.29$ & $3.25 \pm 1.22$ & 0.33 & $-2.835,3.437$ & 0.184 \\
\hline P50-N95lat & $94.8 \pm 9.39$ & $90.85 \pm 10$ & 0.13 & $-19.603,27.503$ & 0.222 \\
\hline N35lat16 & $22.15 \pm 3.4$ & $22.95 \pm 3.88$ & 0.24 & $-11.692,10.092$ & -0.16 \\
\hline N35lat0.8 & $34.4 \pm 8.07$ & $29.35 \pm 5.75$ & 0.002 & $-14.081,24.181$ & 0.024 \\
\hline P50lat16 & $43.35 \pm 2.53$ & $43.8 \pm 3.03$ & 0.34 & $-8.047,7.047$ & -0.181 \\
\hline P50lat0.8 & $52.05 \pm 8.52$ & $50.5 \pm 9.95$ & 0.16 & -24.227, & -0.009 \\
\hline N35-P50amp16 & $5.93 \pm 2.87$ & $5.87 \pm 1.8$ & 0.32 & $-6.708,6.826$ & -0.039 \\
\hline N35-P50amp0.8 & $2.2 \pm 1.53$ & $2.7 \pm 2.2$ & 0.35 & $-5.271,4.257$ & 0.166 \\
\hline PERG ratio (right) & $0.284 \pm 0.183$ & $0.466 \pm 0.314$ & 0.06 & $-0.841,0.713$ & 0.692 \\
\hline PERG ratio (left) & $0.49 \pm 0.175$ & $0.425 \pm 0.255$ & 0.08 & $-0.553,0.667$ & 0.676 \\
\hline
\end{tabular}

*P50-N95amp =P50-N95 amplitude +P50-N95lat = P50-N95 latency ‡N35lat16 = N35 latency with 16 degree check size $\S N 35$ lat $0.8=$ N35 latency with 0.8 degree check size ||P50lat16 = P50 latency with 16 degree check size **P50lat0.8 =P50 latency with 0.8 degree check size †+N35-P50lat $16=$ N35-P50 latency with 16 degree check size $\neq \neq N 35-\mathrm{P} 50$ lat $0.8=\mathrm{N} 35-\mathrm{P} 50$ latency with 16 degree check size $\S \S I C C=$ Intra-class correlation coefficient 


\section{Discussion}

We already know that the conversion rate from untreated $\mathrm{OHT}$ to glaucoma is only $\sim 1 \%$ per year. ${ }^{19}$ Accordingly, most patients with $\mathrm{OHT}$ never have glaucoma and thus do not need treatment. Hence, a method to identify these high risk patients is essential to avoid over treatment. Wanger et al. ${ }^{20}$ reported a significant reduction in the amplitude of PERG in OHT patients. Weinstein GW et al. ${ }^{21}$ found a selective reduction in the second negative wave $(\mathrm{N}-95)$ in glaucomatous patients and concluded that this wave $(\mathrm{N}-95)$ is related to early optic nerve dysfunction. Arai $\mathrm{M}$ et al. ${ }^{12}$ further strengthened this concept when they showed that decrease in the amplitude of the second negative wave (N-95) is a warning sign of development of glaucoma in ocular hypertensives. In our study also we found that the P50-N95 amplitude was statistically significantly less in the OHT group than the controls. However, the difference was not beyond the $95 \%$ limits of test-retest variability of the machine. The previous studies do not mention the test-retest variability of their machines. In addition, the P50lat 16 and the P50lat0.8 was more in the OHT group while theN35-P50amp16 was greater in the normal patients. TheP50lat16 was the only PERG parameter which had a between group difference greater than the retest variability. This makes it a possible candidate for early indicator of glaucoma. The ICC for all PERG parameters in our study showed poor to fair agreement except for the PERG ratio. Bowd et al. ${ }^{22}$ in their study demonstrated a within-trial and between trial ICC of 0.85 and 0.88 respectively. However, they did not calculate the ICC for each PERG parameter as we have done in our study. Bach $\mathrm{M}$ et al..$^{23}$ described that an individual with a large $0.8^{\circ}$ PERG will also have a large $16^{\circ}$ PERG and hence it is useful to compute the PERG ratio to reduce the inter-individual variability. Our study finding is in agreement with this. This low variability for the PERG ratio maybe extremely important while monitoring for change over time. Refractive errors decrease the small check size amplitudes more than large check size amplitudes, mainly due to reduction of the visual acuity. ${ }^{24}$ In our study, we overcame this shortcoming by including only those patients with a best corrected visual acuity $\geq$ 0.8. But, this issue somewhat limits the general applicability of the PERG for early glaucoma detection.

This study has its limitations. We had a relatively small sample size and the PERG measurements were all one time measurements. A better way of doing this study would have been to follow-up all these patients and look for PERG changes over time and amongst those who convert to glaucoma. This would throw light on the predictive accuracy of PERG. This study is currently under way. Another confounder was the fact that treatment of OHT eyes was allowed in our study. Ventura LM et al. ${ }^{25}$ have showed that abnormal PERG recorded in the early stages of glaucoma may often improve after IOP reduction. However, treating eyes with OHT is a real life situation that we encounter very often and hence it was not considered as exclusion criteria. Our current study gives us insight into what PERG parameters would have a low variability which could be used for comparison over time. To the best of our knowledge this is the only study which looks into PERG difference between $\mathrm{OHT}$ and normal patients with calculation of the retest variability of the PERG 
machine. It is extremely important to find parameters with difference outside this range in order to be sure of a real change over time. The increase in the P50 latency has emerged as a new candidate parameter to check for possible conversion of $\mathrm{OHT}$ eyes to glaucoma in future. This needs further confirmation by longitudinal studies with larger sample sizes.

\section{Conclusion}

Increase in P50 latency emerges as a new candidate for early glaucoma indicator from our study in addition to reduction in P50-N95 amplitude which is already known. PERG parameters suffer from high test-retest variability and hence deterioration in PERG recordings should be interpreted with caution. The changes in the PERG parameters need to be greater than the inherent variability of the machine. The variability is lesser for the PERG ratio which maybe more meaningful while monitoring for change over time.

\section{References}

1. Kerrigan-Baumrind LA, Quigley HA, Pease ME, Kerrigan DF, Mitchell RS. Number of ganglion cells in glaucoma eyes compared with threshold visual field tests in the same persons. Invest Ophthalmol Vis Sci 2000;41:741-748.

2. Holder GE. Pattern electroretinography (PERG) and an integrated approach to visual pathway diagnosis. Prog Retin Eye Res 2001;20:531-561.

3. Maffei L, Fiorentini A. Electroretinographic responses to alternating gratings before and after section of the optic nerve. Science 1981;211:953-954.

4. Zrenner E. Physiological basis of the pattern electroretinogram. Prog Retin Res 1989;9:427-464.

5. Ventura LM, Porciatti V. Pattern electroretinogram in glaucoma. Curr Opin Ophthalmol 2006;17:196-202.

6. Pfeiffer N, Tillmon B, Bach M. Predictive value of the pattern electroretinogram in high-risk ocular hypertension. Invest Ophthalmol Vis Sci 1993;34:1710-1715.

7. Walker WM. Ocular hypertension: Follow-up of 109 cases from 1963 to 1974. Trans Ophthalmol Soc UK 1974; 94: 525-534.

8. Porciatti V, Falsini B, Brunori S, Colotto A, Moretti G. Pattern electroretinogram as a function of spatial frequency in ocular hypertension and early glaucoma. Doc Ophthalmol 1987;65:349-355.

9. Bach M, Speidel-Fiaux A. Pattern electroretinogram in glaucoma and ocular hypertension. Doc Ophthalmol 1989;73:173-181.

10. Parisi V, Manni G, Gandolfi SA, Centofanti M, Colacino G, Bucci MG. Visual function correlates with nerve fiber layer thickness in eyes affected by ocular hypertension. Invest Ophthalmol Vis Sci 1999;40:1828-1833.

11. Arai M, Yoshimura N, Sakaue H, Chihara E, Honda Y. A 3-year follow-up study of ocular hypertension by pattern electroretinogram. Ophthalmologica 1993;207:187-195.

12. Gonzalvo Ibanez FJ, Fernandez Tirado FJ, Almarcegui Lafita C, Polo Llorens V, Sanchez Perez A, Honrubia Lopez FM. Predictive value of the pattern-electroretinogram in glaucoma (in Spanish). Arch Soc Esp Oftalmol 2001;76:485-491.

13. Bayer AU, Maag KP, Erb C. Detection of optic neuropathy in glaucomatous eyes with normal standard visual fields using a test battery of short-wavelength automated perimetry and pattern electroretinography. Ophthalmology 2002;109:1350-1361.

14. Ventura LM, Porciatti V, Ishida K, Feuer WJ, Parrish RK 2nd. Pattern electroretinogram abnormality and glaucoma. Ophthalmology 2005;112:10-19.

15. Bach M, Hoffmann MB. Update on the pattern electroretinogram in glaucoma. Optom Vis Sci 2008;85:386-395. 
16. Bach M, Unsoeld AS, Philippin H, Staubach F, Maier P, Walter HS, Bomer TG, Funk J. Pattern ERG as an early glaucoma indicator in ocular hypertension: a long-term, prospective study. Invest Ophthalmol Vis Sci 2006;47:4881-4887.

17. Holder GE, Brigell GM, Hawlina M, Meigen T, Veagan, Bach M. ISCEV standard for clinical pattern electroretinography-2007 update. Doc Ophthalmol 2007;114:111-116.

18. Chapter in a book: Bach M, Hoffmann MB. The origin of the pattern electroretinogram (PERG). In: Heckenlively J, Arden G, editors. Principles and Practice of Clinical Electrophysiology of Vision. Massachusetts: Cambridge MIT Press; 2006. pp.185-196.

19. Kass MA, Heuer DK, Higginbotham EJ, Johnson CA, Keltner JL, Parrish RK 2nd, Wilson MR, Gordon MO. The Ocular Hypertension Treatment Study: a randomized trial determines that topical ocular hypotensive medication delays or prevents the onset of primary open-angle glaucoma. Arch Ophthalmol 2002;120:701-713; discussion 829-830.

20. Wanger $P$, Persson HE. Pattern reversal electroretinogram in ocular hypertension. Doc Ophthalmol 1983;61:27-31.

21. Weinstein GW, Arden GB, Hitchings RA, Ryan S, Calthorpe CM, Odom JV. The pattern ERG in ocular hypertension and glaucoma. Arch Ophthalmol 1988;106(7):923-8.

22. Bowd C, Tafreshi A, Vizzeri G, Zangwill LM, Sample PA, Weinreb RN. Repeatability of pattern electroretinogram measurements using a new paradigm optimized for glaucoma detection. J Glaucoma 2009;18(6):437-442.

23. Bach M. Electrophysiological approaches for early detection of glaucoma. (review) Eur J Ophthalmol 2001;11(suppl 2):41-49.

24. Bach $M$, Mathieu M. Different effect of dioptric defocus vs. lightscatter on the pattern electroretinogram (PERG). Doc Ophthalmol 2004;108:99-106.

25. Ventura LM, Porciatti V. Restoration of retinal ganglion cell function in early glaucoma after intraocular pressure reduction: a pilot study. Ophthalmology 2005;112:20-27. 